\title{
Research on Kinematic Analysis of a Tandem Attitude Control Platform
}

\author{
Xiong Wang ${ }^{1, a^{*}}$ and Yaolong Zhang ${ }^{2, b}$ \\ ${ }^{1,2}$ Yulin University, Yulin, Shanxi, 719000, P.R. China \\ a12296832@qq.com, b2547591586@qq.com
}

Keywords: Attitude control; Angle; Kinematics; Inverse kinematics

\begin{abstract}
Considering shortcomings of existing attitude control platforms, a new directly driven tandem attitude control platform that can bear certain acting force and make accurate and steady operations was designed in this paper. A coordinate system was established by simplifying its structure and its kinematical equation was created and solved. By inputting an angle to different drivers, this tandem attitude control platform can output angle of pitch, rolling angle and course angle accurately and steadily. It was confirmed reliable by the case.
\end{abstract}

\section{Introduction}

Attitude control platform is a platform device that makes the controlled object on the platform keep constant attitude under disturbances in relative to the inertial space or enables the controlled object to adjust under the command according to the given law in relative to the inertial space $[1,2,3]$. It has been widely applied in daily life and military. Among them, the directly driven tandem attitude control platform is highly appreciated for its unique advantages $[4,5,6]$. In some occasions, it couldn't realize the expected performance due to the structural defects and control algorithm defects. Hence, it has very important significance to design a new directly driven tandem attitude control platform that can bear some acting force and make accurate and steady operations $[7,8,9,10]$.

\section{The Tandem Attitude Control Platform}

The tandem attitude control platform is shown in Fig. 1.

(1) The base feet and bed plate support the whole attitude control platform. Some balls are put between the bed plate and the turntable, which can reduce frictions and support the turntable.

(2) There's a 1\# steering engine underneath the turntable to drive the turntable.

(3) A half-cylinder support is installed above the turntable. The 2\# steering engine is fixed on the turntable through a connection to drive the connecting rod rotating with the half-cylinder support.

(4) The 3\# steering engine is installed on the half-cylinder through a connection to drive rotation of the platform where the controlled object is put on.

The controlled object is fixed on the platform and movement of three steering engines can provide the controlled object course angle, angle of pitch and rolling angle.

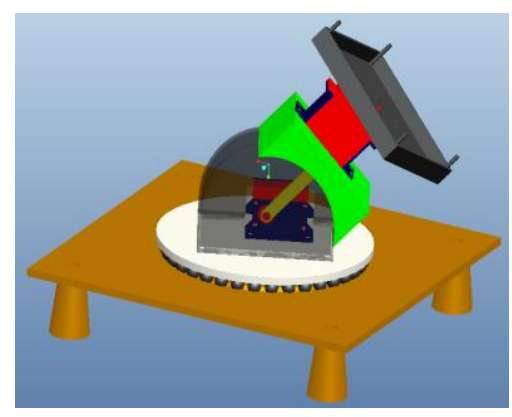

Figure 1. The Tandem Attitude Control Platform 


\section{Kinematical Analysis}

The tandem attitude control platform can be viewed as a multi-rigid-body system composed by a series of connecting rods which are connected by joints. The simplified structure of connecting rod is shown as Fig. 2.

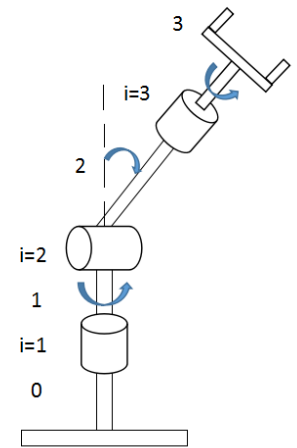

Figure 2. The Simplified Structure of Connecting Rod

The corresponding coordinate system of connecting rod is shown as Fig. 3.

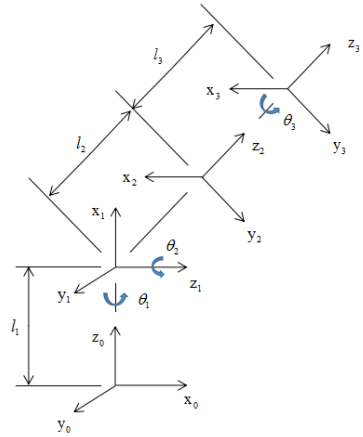

Figure 3. The Corresponding Coordinate System

Combining with actual situations, given length of the connecting rod, parameters of its coordinate system are listed in Table 1, where $\theta_{i}$ is turning angle of the connecting rod, $a_{i}$ is the length of connecting rod, $\alpha_{i}$ is torsional angle of the connecting rod, and $d_{i}$ is the distance between connecting rods.

Table 1 Parameters of connecting rods in the attitude control platform

\begin{tabular}{ccccccc}
\hline$i$ & $\theta_{i}$ & $a_{i}$ & $\alpha_{i}$ & $d_{i}$ & $\cos \alpha_{i}$ & $\sin \alpha_{i}$ \\
\hline 1 & $\theta_{1}$ & $l_{1}=17.5[\mathrm{~mm}]$ & $-90^{\circ}$ & 0 & 0 & -1 \\
2 & $\theta_{2}$ & $l_{2}=107.6[\mathrm{~mm}]$ & $90^{\circ}$ & 0 & 0 & 1 \\
3 & $\theta_{3}$ & $l_{3}=0[\mathrm{~mm}]$ & 0 & 0 & 1 & 0
\end{tabular}

The transformation matrix from the coordinates $\{i\}$ to the homogeneous coordinates $\{i-1\}$ is:

$$
A_{i}=\operatorname{Rot}\left(z_{i-1}, \theta_{i}\right) \operatorname{Trans}\left(0,0, d_{i}\right) \operatorname{Trans}\left(a_{i}, 0,0\right) \operatorname{Rot}\left(x_{i}, \alpha_{i}\right)
$$

Since the attitude control platform finally gets attitude of the end platform which is unrelated with position, the matrix A deletes translation variation and turns into the matrix X, as shown in Eq.1. 
$X_{i}=\operatorname{Rot}\left(z_{i-1}, \theta_{i}\right) \operatorname{Rot}\left(x_{i}, \alpha_{i}\right)=\left[\begin{array}{cccc}\cos \theta_{i} & -\sin \theta_{i} \cos \alpha_{i} & \sin \theta_{i} \sin \alpha_{i} & 0 \\ \sin \theta_{i} & \cos \theta_{i} \cos \alpha_{i} & -\cos \theta_{i} \sin \alpha_{i} & 0 \\ 0 & \sin \alpha_{i} & \cos \alpha_{i} & 0 \\ 0 & 0 & 0 & 1\end{array}\right]$

If $c=\cos$ and $s=\sin$, then,

$$
\begin{gathered}
X_{3}=\left[\begin{array}{cccc}
c_{3} & -s_{3} & 0 & 0 \\
s_{3} & c_{3} & 0 & 0 \\
0 & 0 & 1 & 0 \\
0 & 0 & 0 & 1
\end{array}\right] X_{2}=\left[\begin{array}{cccc}
c_{2} & 0 & s_{2} & 0 \\
s_{2} & 0 & -c_{2} & 0 \\
0 & 1 & 0 & 0 \\
0 & 0 & 0 & 1
\end{array}\right] X_{1}=\left[\begin{array}{cccc}
c_{1} & 0 & -s_{1} & 0 \\
s_{1} & 0 & c_{1} & 0 \\
0 & -1 & 0 & 0 \\
0 & 0 & 0 & 1
\end{array}\right] \\
X_{1} X_{2} X_{3}=\left[\begin{array}{cccc}
c_{1} c_{2} c_{3}-s_{1} s_{3} & -c_{3} s_{1}-c_{1} c_{2} c_{3} & c_{1} s_{2} & 0 \\
c_{1} s_{3}+c_{2} c_{3} s_{1} & c_{1} c_{3}-c_{2} s_{1} s_{3} & s_{1} s_{2} & 0 \\
-c_{3} s_{2} & s_{2} s_{3} & c_{2} & 0 \\
0 & 0 & 0 & 1
\end{array}\right]
\end{gathered}
$$

Matrix $\mathrm{T}$ is expressions of position and attitude of the end platform in relative to the reference coordinate system:

$$
T=\operatorname{Trans}\left(p_{x}, p_{y}, p_{z}\right) \operatorname{Rot}(\kappa, \theta)=\left[\begin{array}{cccc}
n_{x} & o_{x} & a_{x} & p_{x} \\
n_{y} & o_{y} & a_{y} & p_{y} \\
n_{z} & o_{z} & a_{z} & p_{z} \\
0 & 0 & 0 & 1
\end{array}\right]
$$

Since the attitude control platform finally gets attitude of the end platform which is unrelated with position, the matrix $\mathrm{T}$ deletes translation variation and turns into the matrix $\mathrm{Z}$ :

$$
Z=\operatorname{Rot}(\kappa, \theta)=\left[\begin{array}{cccc}
n_{x} & o_{x} & a_{x} & 0 \\
n_{y} & o_{y} & a_{y} & 0 \\
n_{z} & o_{z} & a_{z} & 0 \\
0 & 0 & 0 & 1
\end{array}\right]
$$

Attitude of the end platform is expressed by RPY transformation, as shown in Eq.2.

$$
Z=R P Y(\varphi, \theta, \psi)=\left[\begin{array}{cccc}
c \varphi c \theta & c \varphi s \theta s \psi-s \varphi c \psi & c \varphi s \theta c \psi+s \varphi s \psi & 0 \\
s \varphi c \theta & s \varphi s \theta s \psi+c \varphi c \psi & s \varphi s \theta c \psi-c \varphi s \psi & 0 \\
-s \theta & c \theta s \psi & c \theta c \psi & 0 \\
0 & 0 & 0 & 1
\end{array}\right]
$$

Kinematical Equation of the Attitude Control Platform. Through premultiplication of (2) by $\operatorname{Rot}(z, \varphi)^{-1}$, it gets:

$$
\left[\begin{array}{cccc}
n_{x} c \varphi+n_{y} s \varphi & o_{x} c \varphi+o_{y} s \varphi & a_{x} c \varphi+a_{y} s \varphi & 0 \\
-n_{x} s \varphi+n_{y} c \varphi & -o_{x} s \varphi+o_{y} c \varphi & -a_{x} s \varphi+a_{y} c \varphi & 0 \\
n_{z} & o_{z} & a_{z} & 0 \\
0 & 0 & 0 & 1
\end{array}\right]=\left[\begin{array}{cccc}
c \theta & -s \theta c \psi & s \theta c \psi & 0 \\
0 & c \psi & -s \psi & 0 \\
-s \theta & c \theta s \psi & c \theta c \psi & 0 \\
0 & 0 & 0 & 1
\end{array}\right]
$$

The corresponding terms are equal and then, the angle can be solved, as shown in Eq.3. 


$$
\left\{\begin{array}{l}
\varphi=\arctan \left(\frac{n_{y}}{n_{x}}\right) \\
\theta=\arctan \left(\frac{-n_{z}}{n_{x} c \varphi+n_{y} s \varphi}\right) \\
\psi=\arctan \left(\frac{a_{x} s \varphi-a_{y} c \varphi}{-o_{x} s \varphi+o_{y} c \varphi}\right)
\end{array}\right.
$$

According to $Z=X_{1} X_{2} X_{3}$, it can change into:

$$
\left\{\begin{array}{l}
n_{x}=c_{1} c_{2} c_{3}-s_{1} s_{3} \\
n_{y}=c_{1} s_{3}+c_{2} c_{3} s_{1} \\
n_{z}=-c_{3} s_{2} \\
o_{x}=-c_{3} s_{1}-c_{1} c_{2} c_{3} \\
o_{y}=c_{1} c_{3}-c_{2} s_{1} s_{3} \\
o_{z}=s_{2} s_{3} \\
a_{x}=c_{1} s_{2} \\
a_{y}=s_{1} s_{2} \\
a_{z}=c_{2}
\end{array}\right.
$$

Solving of the Kinematical Equation of the Attitude Control Platform. The attitude of the end platform is: $Z=X_{1} X_{2} X_{3}$.

Unfold two sides of the equation and it gets:

$$
\left[\begin{array}{cccc}
c \varphi c \theta & c \varphi s \theta s \psi-s \varphi c \psi & c \varphi s \theta c \psi+s \varphi s \psi & 0 \\
s \varphi c \theta & s \varphi s \theta s \psi+c \varphi c \psi & s \varphi s \theta c \psi-c \varphi s \psi & 0 \\
-s \theta & c \theta s \psi & c \theta c \psi & 0 \\
0 & 0 & 0 & 1
\end{array}\right]=\left[\begin{array}{cccc}
c_{1} c_{2} c_{3}-s_{1} s_{3} & -c_{3} s_{1}-c_{1} c_{2} c_{3} & c_{1} s_{2} & 0 \\
c_{1} s_{3}+c_{2} c_{3} s_{1} & c_{1} c_{3}-c_{2} s_{1} s_{3} & s_{1} s_{2} & 0 \\
-c_{3} s_{2} & s_{2} s_{3} & c_{2} & 0 \\
0 & 0 & 0 & 1
\end{array}\right]
$$

The corresponding terms are equal,

$$
\left\{\begin{array}{l}
c \theta c \psi=c_{2} \\
-s \theta=-c_{3} s_{2} \\
c \varphi s \theta c \psi+s \varphi s \psi=c_{1} s_{2}
\end{array}\right.
$$

So the rotation angle of the joint can be solved, as shown in Eq.4.

$$
\left\{\begin{array}{l}
\theta_{1}=\arccos \left\{\frac{c \varphi s \theta c \psi+s \varphi s \psi}{\sin [\arccos (c \theta c \psi)]}\right\} \\
\theta_{2}=\arccos (c \theta c \psi) \\
\theta_{3}=\arccos \left\{\frac{s \theta}{\sin [\arccos (c \theta c \psi)]}\right\}
\end{array}\right.
$$

Many results are gained and chose one group of solutions as the solutions of the equations. $\theta_{1}$ ranges between $-180^{\circ} \sim 180^{\circ} ; \theta_{2}$ and $\theta_{3}$ range between $-90^{\circ} \sim 90^{\circ} ; \varphi$ and $\theta$ range between $-90^{\circ} \sim 90^{\circ}$; and $\psi$ ranges between $-180^{\circ} \sim 180^{\circ}$.

\section{Experimental Verification}

To verify feasibility of the design, one attitude is chosen as the input, thus getting $\theta_{1}, \theta_{2}$ and $\theta_{3}$ of different steering engines. 
Let $\theta=30^{\circ}, \varphi=30^{\circ}$ and $\psi=30^{\circ}$, Substitute them into (4) and it gains:

$$
\left\{\begin{array}{l}
\theta_{1}=19^{\circ} \\
\theta_{2}=41^{\circ} \\
\theta_{3}=41^{\circ}
\end{array}\right.
$$

This indicates that the $1 \#$ steering engine makes counterclockwise rotation by $19^{\circ}$, the $2 \#$ steering engine makes counterclockwise rotation by $41^{\circ}$ and the $3 \#$ steering engine makes counterclockwise rotation by $41^{\circ}$. It is confirmed that the expected attitude could be gained in this way.

\section{Summary}

Considering the weak vertical support of directly driven tandem attitude control platform, this paper keeps the simple output of attitude control angles and makes some structural innovations. The kinematics of tandem attitude control platform are analyzed. Finally, the case study confirms that the designed attitude control platform can achieve expected attitude angles according to rotating angles of driving motors, meeting the design requirements.

\section{Acknowledgements}

This work was financially supported by the university-industry cooperation projects of Yulin science and technology Bureau (2014cxy-08-02).

\section{References}

[1] G.D Li, J.C Cao, B.Li and H.F. Li: Advanced Materials Research, Vol. 2385 (2013) No. 694, p. 1512-1518.

[2] M. Hanmandlu, G. Himani and D.P. Kothari: IETE Journal of Research, Vol.53 (2007) No. 2, p.119.

[3] M. Deepika, J. Shailendra and A. Gayatri: Journal of Research,Vol.54 (2008) No. 6, p.421.

[4] T. MENG and S. MATUNAGA: TRANSACTIONS OF THE JAPAN SOCIETY FOR AERONAUTICAL AND SPACE SCIENCES, Vol.54 (2011) No.185, p.198-204.

[5] J. Love and M. Bunch: Chemical Engineering Research and Design, Vol.76 (1998) No.8, p.973-979.

[6] S.E. LYSHEVSKI: Automatica, Vol.34 (1998) No.10, p.1231-1238.

[7] A.G. Parlos and O.T. Rais: Control Engineering Practice, Vol.8 (2000) No.8, p.921-936.

[8] D.C. Liaw, Y.W. Liang and C.C. Cheng: Journal of Dynamic Systems, Measurement, and Control, Vol.122 (2000) No.4, p.663.

[9] L. TIAN and S. XU: Chinese Journal of Aeronautics, Vol.25 (2012) No.3, p.437-445.

[10] V.Azhmyakov, A.E. Gil and M.V. Basin.: Kybernetika, Vol.50 (2014) No.1, p.5-18. 